OPEN ACCESS

Edited by:

John R. Battista,

Louisiana State University, USA

Reviewed by:

Seong Woon Roh,

Korea Basic Science Institute, South

Korea

Asaf Levy,

Lawrence Berkeley National Lab, USA

*Correspondence:

Longbiao Guo

guolongb@mail.hz.zj.cn

${ }^{\dagger}$ These authors have contributed equally to this work.

Specialty section: This article was submitted to Evolutionary and Genomic Microbiology,

a section of the journal

Frontiers in Microbiology

Received: 02 August 2016 Accepted: 19 October 2016 Published: 08 November 2016

Citation:

Lin H, Hu S, Liu R, Chen P, Ge C,

Zhu $B$ and Guo L (2016) Genome Sequence of Pseudomonas koreensis CRSO5-R5, an Antagonistic Bacterium Isolated from Rice Paddy Field.

Front. Microbiol. 7:1756.

doi: 10.3389/fmicb.2016.01756

\section{Genome Sequence of Pseudomonas koreensis CRS05-R5, an Antagonistic Bacterium Isolated from Rice Paddy Field}

\author{
Haiyan Lin ${ }^{1,2 \dagger}$, Shikai Hu ${ }^{1 \dagger}$, Ruifang Liu ${ }^{1,2}$, Ping Chen ${ }^{1}$, Changwei Ge ${ }^{1}$, Bo Zhu ${ }^{3}$ and \\ Longbiao Guo ${ }^{1 *}$ \\ ${ }^{1}$ State Key Lab for Rice Biology, China National Rice Research Institute, Hangzhou, China, ${ }^{2}$ Agricultural Genomes Institute at \\ Shenzhen, Chinese Academy of Agricultural Sciences, Shenzhen, China, ${ }^{3}$ Zhejiang Province Key Laboratory of Plant \\ Secondary Metabolism and Regulation, College of Life Science, Zhejiang Sci-Tech University, Hangzhou, China
}

Keywords: biocontrol, Pseudomonas koreensis, PacBio, secondary metabolism, unique gene

\section{INTRODUCTION}

Pseudomonas koreensis, a new nominated Gram-negative bacterium was first reported and isolated from Korean agricultural soil (Kwon et al., 2003). CRS05-R5 (first reported as Pseudomonas sp.), which showed biocontrol ability against Sitophilus oryzae and Acidovorax avenae subsp. avenae (Liu et al., 2014), was first isolated from the rice rhizosphere in Heilongjiang province and reported in 2003 (Xie et al., 2003). Except for that, this species has been reported to produce the biosurfactant, which has biocontrol ability against Phytophthora infestans and Pythium ultimum (Hultberg et al., 2010a,b). These interesting features raise our attention on CRS05-R5. Recently, we sequenced the 16S rRNA sequence from CRS05-R5 and built the phylogenetic tree (Figure S1). Based on that, we confirmed that CRS05-R5 should be classified as P. koreensis. However, only one genome was sequenced (D26) and no detailed analysis was performed on this species. In this case, we did whole-genome sequencing on CRS05-R5, and tried to reveal the possible mechanism behind its antagonistic ability.

\section{METHODS}

\section{Genomic DNA Isolation}

Single colony of CRS05-R5 was inoculated into $5 \mathrm{ml} \mathrm{NB}$ (Nutrient Broth, BD, USA) at $30^{\circ} \mathrm{C}$ with $180 \mathrm{rpm}$ vigorous shaking. $2.5 \mathrm{ml}$ of culture broth was used to isolate the genomic DNA. DNA was extracted by Wizard Genomic DNA Purification Kit (Promega, Madison, WI, USA). The quality of purified genomic DNA was tested by using NanoDrop 2000 UV-Vis spectrophotometer (Thermo Scientific, MA, USA) and Qubit 2.0 fluorometer (Life Technologies, MA, USA), respectively.

\section{Whole Genome Sequencing and Annotation}

Whole genome sequencing of CRS05-R5 was carried out by using PacBio RS II platform. Six hundred Megabytes raw data was obtained with 100X coverage. After quality control, genome assembly was de novo assembled using HGAP assembly protocol, which is available with the SMRT Analysis packages and accessed through the SMRT Analysis Portal version 2.1. Genome annotation was later done by using RAST annotation system (Overbeek et al., 2014). The completeness of the assembled genome was tested by CheckM with default parameters (Parks et al., 2015). In addition, GO and COG programs were used to do further functional analysis of all annotated ORFs (Ashburner et al., 2000; Tatusov et al., 2000). Specifically, since antagonistic bacteria, especially 
fluorescent pseudomonads, usually compete with other microorganisms by using secondary metabolism (Haas and Défago, 2005), genes, which are predicted to be involved in secondary metabolism were compared with DoBISCUIT database (Ichikawa et al., 2013). The circular genome map of CRS05-R5 including all predicted ORFs with COG functional assignments, rRNA, tRNA, G+C content, and GC skew information were generated using Circos (Krzywinski et al., 2009), as shown in Figure S2.

\section{Genome Comparison}

Genome comparison among CRS05-R5 and other fully sequenced Pseudomonas genomes were carried out by using ANI (average nucleotide identity) and AF (alignment fraction), which were calculated by ANIcalculator (Varghese et al., 2015), and Circos (Krzywinski et al., 2009). In order to find out the unique genes in CRS05-R5, all the fully sequenced Pseudomonas genomes were downloaded from NCBI (58 genomes). The protein sequences from CRS05-R5 were compared with the protein sequences from these 58 genomes with $40 \%$ identity as cutoff. The protein sequences which cannot find any homologs in these 58 genomes were classified as unique genes.

\section{Direct Link to Deposited Data and Information to Users}

This strain has been deposited in CGMCC with deposit number 1.15630. This genome sequencing project has been deposited at DDBJ/EMBL/GenBank under the Accession Number CP015852. The BioProject designation for this project is PRJNA322127.

\section{INTERPRETATION OF DATA SET}

\section{General Genome Sequence Property}

The total size of the genome is 5,991,224 bp and has a $\mathrm{G}+\mathrm{C}$ content of $60.6 \%$. The completeness and contamination of this genome is $99.86 \%$ and $0.05 \%$ after running the CheckM. These results indicate the high quality of assembled genome. A total of 5352 CDSs were predicted. Of these, 3832 could be assigned to a COG number. The most abundant COG category was "General function prediction only" (581 proteins) followed by "Signal transduction mechanisms" (547 proteins), "Amino acid transport and metabolism" (539 proteins), "Transcription” (448 proteins), and "Function unknown" (302 proteins). In addition, 82 RNAs including rRNA and tRNA were identified. All the genomic information was shown in Table $\mathbf{1}$.

\section{Secondary Metabolism in CRS05-R5}

Biosurfactants are amphiphilic compounds produced by microorganisms (Mulligan, 2005). This compound, which is produced by Pseudomonas spp., has been widely used in biocontrol (Debode et al., 2007). In CRS05-R5 genome, more than 800 genes are predicted to be involved in secondary metabolism by comparing with DoBISCUIT database (Ichikawa et al., 2013). Interestingly, we found one gene cluster, which is annotated as cyclic lipopeptides (CLPs), exists in CRS05-R5 $(\operatorname{arf} A B C)$. CLP is a kind of biosurfactant, and has been proved to be important in antagonistic Pseudomonas sp. (Raaijmakers
TABLE 1 | Genome statistics.

\begin{tabular}{lc}
\hline Features & Value \\
\hline Genome size(bp) & $5,991,224$ \\
Contig numbers & 1 \\
G+C \% & 60.6 \\
Protein-coding genes & 5352 \\
Protein with known function & 4363 \\
tRNA number & 76 \\
rRNA number & 6 \\
ncRNA number & 75 \\
Genes with signal peptides & 573 \\
Genes with transmembrane helices & 1187 \\
\hline
\end{tabular}

et al., 2006). This information indicates that CRS05-R5 can be used as biocontrol agent.

\section{Genome Comparison}

Strikingly, we found even the ANI value between CRS05-R5 and P. koreensis D26 is only 92.5\% (Figure S3). Also, the highest AF value is only $74.2 \%$ (Table S1). Indeed, 631 CDSs were predicted to be unique genes existing in CRS05-R5 genome. These results indicate the huge genome diversity among Pseudomonas strains, which is consistent with previous findings (Loper et al., 2012). Circos result found many unique genes in CRS05-R5 (Figure 1). To better understand the features of these genes, Pseudomonas database was used to deeply annotate these genes (Winsor et al., 2011). Except for a lot of hypothetical proteins, we found one gene cluster encoding fimbrial associated proteins only existing in CRS05-R5 (A8L59_09240-A8L59_09310). These genes have been reported to be critical for the initial stage of biofilm development (Wei and Ma, 2013). Except for this gene cluster, we also found three rhs genes exist as the unique genes in CRS05-R5 (A8L59_00750, A8L59_09890, and A8L59_11585). These genes have been found to be linked to the second type VI secretion cluster in P. aeruginosa (Jones et al., 2014). Also, these genes have been reported to mediate intercellular competition (Koskiniemi et al., 2013). More strikingly, one rhs gene is located in a unique gene cluster (A8L59_11555A8L59_11600). Although, most of the genes are hypothetical proteins, we may infer that this cluster maybe related with secretions. Indeed, by searching with TMHMM Server v. 2.0, which is a transmembrane helices prediction database (http://www.cbs.dtu.dk/services/TMHMM/), we found that 3 genes (A8L59_11560, A8L59_11565, and A8L59_11600) have predicted transmembrane helices. Since biofilm and intercellular competition are very important for bacterial survival and adaptation, we infer that these genes may confer some fitness advantages for CRS05-R5 on the root of Oryza sativa. Also, we found one unique gene cluster (A8L59_18500-A8L59_18575), which encodes wbpL and other glycosyl transferase. These genes have been confirmed to be important in lipopolysaccharide formation (Rocchetta et al., 1998). These compounds have been proved to be important in plant roots colonization (Duijff et al., 1997) as well as induction of systemic resistance against some 


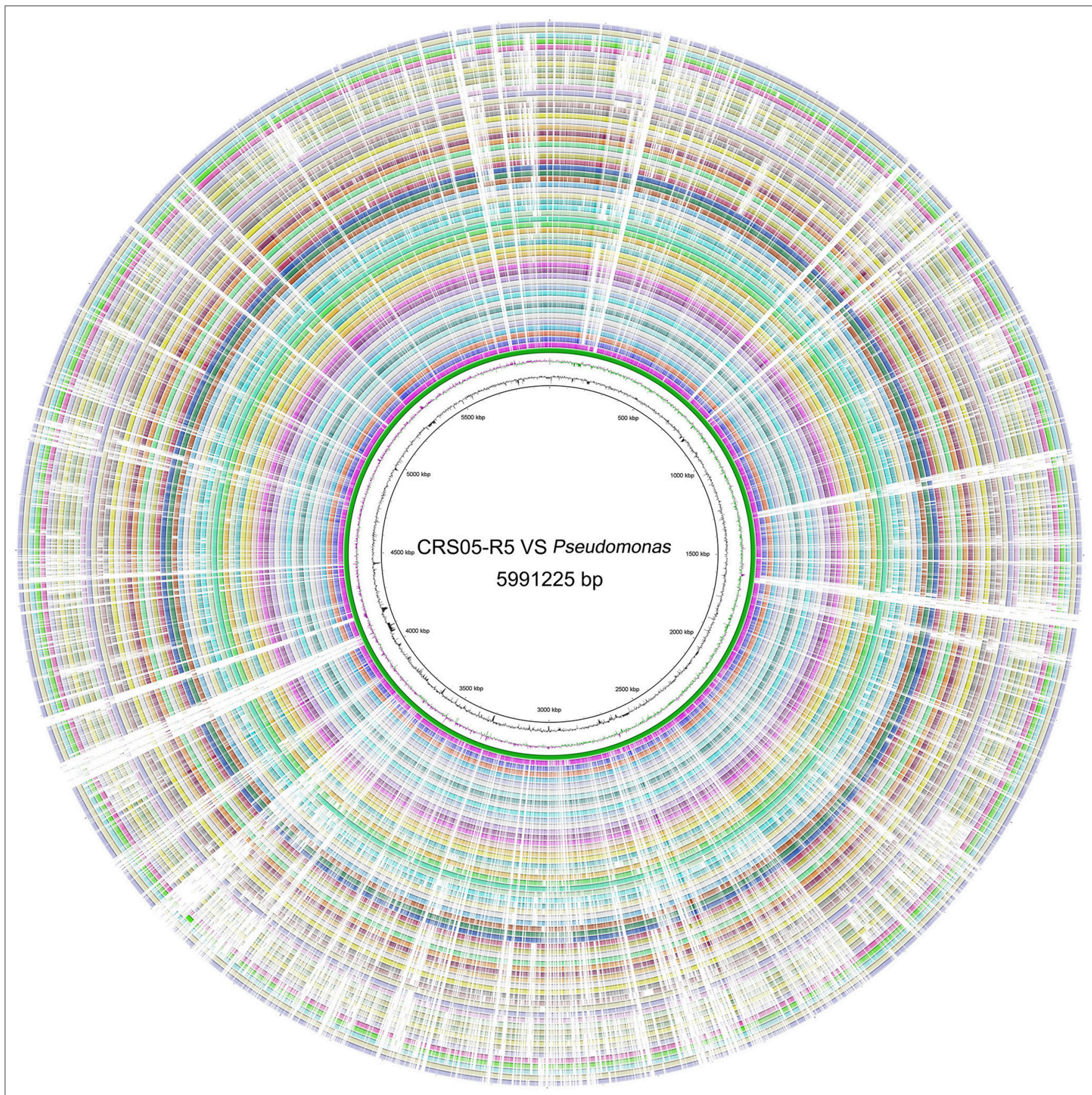

FIGURE 1 | Circular map of the chromosome of $P$. koreensis CRS05-R5 and other fully sequenced $P$ seudomonas genomes. The tracks from the inside to outside: GC Content, GC Skew, P. koreensis CRS05-R5, P. koreensis D26, P. aeruginosa B136-33, P. aeruginosa c7447 m, P. aeruginosa DK2, $P$. aeruginosa LES431, $P$. aeruginosa LESB58, $P$. aeruginosa M18, $P$. aeruginosa MTB-1, $P$. aeruginosa NCGM2.S1, $P$. aeruginosa PA1, $P$. aeruginosa PA1R, $P$. aeruginosa PA7, $P$. aeruginosa PAO1-VE13, P. aeruginosa PAO1-VE2, P. aeruginosa PAO1, P. aeruginosa PAO581, P. aeruginosa RP73, P. aeruginosa SCV20265, P. aeruginosa UCBPP-PA14, $P$. denitrificans ATCC 13867, P. entomophila L48, P. fluorescens A506, P. fluorescens Pfo-1, P. fluorescens SBW25, P. fulva 12-X, P. mendocina NK-01, P. mendocina ymp, P. monteilii SB3078, P. monteilii SB3101, P. poae RE1-1-14, P. protegens CHAO, P. protegens Pf-5, P. putida DOT-T1E, P. putida F1, P. putida GB-1, P. putida H8234, P. putida HB3267, P. putida KT2440, P. putida NBRC 14164, P. putida ND6, P. putida S16, P. putida W619, P. resinovorans NBRC 106553, Pseudomonas sp. TKP, Pseudomonas sp. UW4, Pseudomonas sp. VLB120, P. stutzeri A1501, P. stutzeri ATCC 17588, P. stutzeri CCUG 29243, P. stutzeri DSM 10701, P. stutzeri DSM 4166, P. stutzeri RCH2, P. syringae pv. phaseolicola 1448A, P. syringae pv. syringae B728a, P. syringae pv. tomato DC3000, P. brassicacearum subsp. Brassicacearum NFM421, P. fluorescens F113. 
plant pathogens (Leeman et al., 1995). All these results strongly indicate the biocontrol potential of CRS05-R5 strain.

\section{AUTHOR CONTRIBUTIONS}

HL performed the experiments and write the manuscript. $\mathrm{SH}$ and RL helped in data analysis. PC, CG, and BZ coordinated the work and drafted the manuscript. LG conceived the work.

\section{FUNDING}

This work was supported by grants from the Natural Science Fund of China (grant No. 31521064), and the Chinese 973 Program (2013CBA01405).

\section{ACKNOWLEDGMENTS}

We thank Dr. Weijie Song for sequencing at Biology Technology Company of Guhe.

\section{REFERENCES}

Ashburner, M., Ball, C. A., Blake, J. A., Botstein, D., Butler, H., Cherry, J. M., et al. (2000). Gene ontology: tool for the unification of biology. Nat. Genet. 25, 25-29. doi: $10.1038 / 75556$

Debode, J., De Maeyer, K., Perneel, M., Pannecoucque, J., De Backer, G., and Höfte, M. (2007). Biosurfactants are involved in the biological control of Verticillium microsclerotia by Pseudomonas spp. J. Appl. Microbiol. 103, 1184-1196. doi: 10.1111/j.1365-2672.2007.03348.x

Duijff, B. J., Gianinazzi-Pearson, V., and Lemanceau, P. (1997). Involvement of the outer membrane lipopolysaccharides in the endophytic colonization of tomato roots by biocontrol Pseudomonas fluorescens strain WCS417r. New Phytol. 135, 325-334. doi: 10.1046/j.1469-8137.1997.00646.x

Haas, D., and Défago, G. (2005). Biological control of soil-borne pathogens by fluorescent pseudomonads. Nat. Rev. Microbiol. 3, 307-319. doi: 10.1038/ nrmicrol129

Hultberg, M., Alsberg, T., Khalil, S., and Alsanius, B. (2010a). Suppression of disease in tomato infected by Pythium ultimum with a biosurfactant produced by Pseudomonas koreensis. Biocontrol 55, 435-444. doi: 10.1007/s10526-0099261-6

Hultberg, M., Bengtsson, T., and Liljeroth, E. (2010b). Late blight on potato is suppressed by the biosurfactant-producing strain Pseudomonas koreensis 2.74 and its biosurfactant. Biocontrol 55, 543-550. doi: 10.1007/s10526-0109289-7

Ichikawa, N., Sasagawa, M., Yamamoto, M., Komaki, H., Yoshida, Y., Yamazaki, S., et al. (2013). DoBISCUIT: a database of secondary metabolite biosynthetic gene clusters. Nucleic Acids Res. 41, D408-D414. doi: 10.1093/nar/gks1177

Jones, C., Hachani, A., Manoli, E., and Filloux, A. (2014). An rhs gene linked to the second type vi secretion cluster is a feature of the Pseudomonas aeruginosa strain PA14. J. Bacteriol. 196, 800-810. doi: 10.1128/JB.00863-13

Koskiniemi, S., Lamoureux, J. G., Nikolakakis, K. C., t'Kint de Roodenbeke, C., Kaplan, M. D., Low, D. A., et al. (2013). Rhs proteins from diverse bacteria mediate intercellular competition. Proc. Natl. Acad. Sci. U.S.A. 110, 7032-7037. doi: 10.1073/pnas.1300627110

Krzywinski, M., Schein, J., Birol, I., Connors, J., Gascoyne, R., Horsman, D., et al. (2009). Circos: an information aesthetic for comparative genomics. Genome Res. 19, 1639-1645. doi: 10.1101/gr.092759.109

Kwon, S. W., Kim, J. S., Park, I. C., Yoon, S. H., Park, D. H., Lim, C. K., et al. (2003). Pseudomonas koreensis sp. nov., Pseudomonas umsongensis sp. nov. and Pseudomonas jinjuensis sp. nov., novel species from farm soils in Korea. Int. J. Syst. Evol. Microbiol. 53, 21-27. doi: 10.1099/ijs.0.02326-0

\section{SUPPLEMENTARY MATERIAL}

The Supplementary Material for this article can be found online at: http://journal.frontiersin.org/article/10.3389/fmicb. 2016.01756/full\#supplementary-material

Table S1 | The fraction of orthologous genes (Alignment Fraction, AF) value between CRS05-R5 and other Pseudomonas genomes.

Figure S1 | Phylogenetic relationships based on 16S rRNA gene sequences were determined by the neighbor-joining method with the program package MEGA 6.0 (Tamura et al., 2013). Bootstrap confidence values were obtained using 1000 resamplings. The tree shows the positions of strains CRS05-R5 and other selected Pseudomonas strains. Numbers at nodes indicate percentages of occurrence in 1000 bootstrapped trees; only values $>50 \%$ are shown. Bar, 0.005 substitutions per site.

Figure S2 | Graphical map of the chromosome genome of $\boldsymbol{P}$. koreensis CRS05-R5. From the outside to the center: genes on forward stand and Genes on reverse strand (color by COG categories), RNA genes (tRNAs green, rRNAs red, ncRNAs black), GC content, GC skew.

Figure S3 | Heatmap of Average Nucleotide Identity (ANI) between CRS05-R5 and all the other sequenced Pseudomonas genomes.

Leeman, M., Van Pelt, J., Den Ouden, F., Heinsbroek, M., Bakker, P., and Schippers, B. (1995). Induction of systemic resistance against Fusarium wilt of radish by lipopolysaccharides of Pseudomonas fluorescens. Phytopathology 85, 1021-1027. doi: 10.1094/Phyto-85-1021

Liu, H., Yang, C. L., Ge, M. Y., Ibrahim, M., Li, B., Zhao, W. J., et al. (2014). Regulatory role of tetR gene in a novel gene cluster of Acidovorax avenae subsp. avenae RS-1 under oxidative stress. Front. Microbiol. 5:547. doi: 10.3389/fmicb.2014.00547

Loper, J. E., Hassan, K. A., Mavrodi, D. V., Davis, E. W. II, Lim, C. K., Shaffer, B. T., et al. (2012). Comparative genomics of plant-associated Pseudomonas spp.: insights into diversity and inheritance of traits involved in multitrophic interactions. PLoS Genet. 8:e1002784. doi: 10.1371/journal.pgen.10 02784

Mulligan, C. N. (2005). Environmental applications for biosurfactants. Environ. Pollut. 133, 183-198. doi: 10.1016/j.envpol.2004.06.009

Overbeek, R., Olson, R., Pusch, G. D., Olsen, G. J., Davis, J. J., Disz, T., et al. (2014). The SEED and the Rapid Annotation of microbial genomes using Subsystems Technology (RAST). Nucleic Acids Res. 42, D206-D214. doi: $10.1093 /$ nar/gkt 1226

Parks, D. H., Imelfort, M., Skennerton, C. T., Hugenholtz, P., and Tyson, G. W. (2015). CheckM: assessing the quality of microbial genomes recovered from isolates, single cells, and metagenomes. Genome Res. 25, 1043-1055. doi: $10.1101 /$ gr.186072.114

Raaijmakers, J. M., de Bruijn, I., and de Kock, M. J. D. (2006). Cyclic lipopeptide production by plant-associated Pseudomonas spp.: diversity, activity, biosynthesis, and regulation. Mol. Plant. Microbe Interact. 19, 699-710. doi: 10.1094/MPMI-19-0699

Rocchetta, H. L., Burrows, L. L., Pacan, J. C., and Lam, J. S. (1998). Three rhamnosyltransferases responsible for assembly of the A-band D-rhamnan polysaccharide in Pseudomonas aeruginosa: a fourth transferase, $\mathrm{WbpL}$, is required for the initiation of both A-band and B-band lipopolysaccharide synthesis. Mol. Microbiol. 28, 1103-1119. doi: 10.1046/j.1365-2958. 1998.00871.x

Tamura, K., Stecher, G., Peterson, D., Filipski, A., and Kumar, S. (2013). MEGA6: molecular evolutionary genetics analysis version 6.0. Mol. Biol. Evol. 30, 2725-2729. doi: 10.1093/molbev/mst197

Tatusov, R. L., Galperin, M. Y., Natale, D. A., and Koonin, E. V. (2000). The COG database: a tool for genome-scale analysis of protein functions and evolution. Nucleic Acids Res. 28, 33-36. doi: 10.1093/nar/28.1.33

Varghese, N. J., Mukherjee, S., Ivanova, N., Konstantinidis, K. T., Mavrommatis, K., Kyrpides, N. C., et al. (2015). Microbial species delineation using whole 
genome sequences. Nucleic Acids Res. 43, 6761-6771. doi: 10.1093/nar/ gkv657

Wei, Q., and Ma, L. Z. (2013). Biofilm matrix and its regulation in Pseudomonas aeruginosa. Int. J Mol. Sci. 14, 20983-21005. doi: 10.3390/ijms141020983

Winsor, G. L., Lam, D. K., Fleming, L., Lo, R., Whiteside, M. D., Nancy, Y. Y., et al. (2011). Pseudomonas genome database: improved comparative analysis and population genomics capability for Pseudomonas genomes. Nucleic Acids Res. 39, D596-D600. doi: 10.1093/nar/gkq869

Xie, G. L., Soad, A., Swings, J., and Mew, T. (2003). Diversity of Gram negative bacteria antagonistic against major pathogens of rice from rice seed in the tropic environment. J. Zhejiang Univ. Sci. A 4, 463-468. doi: 10.1631/jzus.2003.0463
Conflict of Interest Statement: The authors declare that the research was conducted in the absence of any commercial or financial relationships that could be construed as a potential conflict of interest.

Copyright (c) $2016 \mathrm{Lin}, \mathrm{Hu}, \mathrm{Liu}, \mathrm{Chen}, \mathrm{Ge}, \mathrm{Zhu}$ and Guo. This is an open-access article distributed under the terms of the Creative Commons Attribution License (CC BY). The use, distribution or reproduction in other forums is permitted, provided the original author(s) or licensor are credited and that the original publication in this journal is cited, in accordance with accepted academic practice. No use, distribution or reproduction is permitted which does not comply with these terms. 